\title{
High Resolution MIMO-HFSWR Radar Using Sparse Frequency Waveforms
}

\author{
Guohua WANG, Yilong LU \\ School of Electrical and Electronic Engineering, Nanyang Technological University, Singapore \\ E-mail: \{wang0330,eylu\}@ntu.edu.sg \\ Received April 21, 2009; revised May 12, 2009; accepted May 15, 2009
}

\begin{abstract}
In high frequency surface wave radar (HFSWR) applications, range and azimuth resolutions are usually limited by the bandwidth of waveforms and the physical dimension of the radar aperture, respectively. In this paper, we propose a concept of multiple-input multiple-output (MIMO) HFSWR system with widely separated antennas transmitting and receiving sparse frequency waveforms. The proposed system can overcome the conventional limitation on resolutions and obtain high resolution capability through this new configuration. Ambiguity function (AF) is derived in detail to evaluate the basic resolution performance of this proposed system. The advantages of the system of fine resolution and low peak sidelobe level (PSL) are demonstrated by the AF analysis through numerical simulations. The impacts of Doppler effect and the geometry configuration are also studied.
\end{abstract}

Keywords: MIMO, HFSWR, Radar, Sparse Frequency Waveform

\section{Introduction}

HIGH frequency surface wave radar (HFSWR) is a low-cost radar system that adopts vertically polarized high frequency electromagnetic signals which propagate along the ocean surface. A preferable property of HFSWR is that it can detect and track ship and aircraft targets beyond the horizon. Due to this reason, HFSWR has a wide range of applications in both civil and military fields. For conventional HFSWR systems, its range resolution is highly restricted by the bandwidth of available clear channels in a congested spectrum environment $[1,2]$, while the azimuth resolution is also constrained by the physical dimension of the radar antenna aperture.

Multiple-input multiple-output (MIMO) radar is now getting much intention for various applications such as detection, estimation, and imaging etc. MIMO radar can transmit at transmitters multiple waveforms that are dividual at the receivers so that it can obtain more degrees of freedom compared with conventional radars that transmit single waveform [3-6]. With widely distributed antennas, angular diversity can be fully achieved to compete with the target scintillations $[7,8]$. Meanwhile, MIMO radars with widely distributed antennas can gain high resolution by coherent processing [8]. Like distrib- uted MIMO radar, a single-input multiple-output (SIMO) radar system with sparse coherent receiving aperture can also achieve high resolution on the order of one wavelength with limited bandwidth as reported in [9].

Sparse frequency waveform problem has been studied in $[10,11]$ and literatures therein. For HFSWR, sparse frequency waveform can provide large flexibility to choose clear channels and thereby reduce interferences from assigned channels. Motivated by the potential benefits from sparse frequency waveforms and high resolution capacity of coherent multistatic radar and MIMO radar systems, we in this paper propose a novel MIMO-HFSWR using sparse frequency waveforms to break down the limitation on range and azimuth resolutions of conventional HFSWR. Ambiguity Function (AF) is derived in detail and fully investigated in this paper to analyze the performance of the proposed system. Unlike that of paper [8], we take Doppler effect in the AF for analysis. Through $\mathrm{AF}$ analysis it is demonstrated that this system has high flexibility in operation and attractive improvement on resolution in the restricted geographical condition as well as the congested spectrum environment. In particular, by using the widely separated antennas, it abates the aperture limitation as well as the rigorous land requirement successfully. In addition, by using sparse 
frequency waveform it not only takes more clear channels into use to compete with co-channel interference but also reduces the peak sidelobe level (PSL).

The reminder of this paper is organized as follows. The AF of the proposed MIMO-HFSWR using sparse frequency waveforms is derived in Section 2. Based on $\mathrm{AF}$ analysis and simulations the system is evaluated in terms of resolution capacity and PSL performance, with zero Doppler frequency in Section 3 and under the factors of geometric configurations and Doppler effects in Section 4. Finally, conclusions and future work are outlined in Section 5.

\section{Ambiguity Function of MIMO-HFSWR System Using Sparse Frequency Waveforms}

Ambiguity function is an important tool in conventional radar analysis as it shows radar's inherent capacity of discriminating targets associated with different time delay and Doppler frequency. Thus, in this paper, we also employ AF to evaluate the performance of the proposed system.

The proposed MIMO-HFSWR system consists of $M$ transmitters transmitting $M$ waveforms and $N$ receivers. Each transmitter is assigned a distinct channel with starting frequency $f_{m}, m=1,2, \ldots, M$. Thus, collectively, the transmitting waveforms will have a sparse spectrum, because which we call the transmitting waveforms sparse frequency waveform. All antennas are arbitrarily located with mutual separation distance larger than several wavelengths in a 3-dimensional space. Figure 1 shows the system configuration, where $R_{n}$ and $T_{m}$ refer to the $n$-th receiver and the $m$-th transmitter, respectively. Each transmitter and each receiver are located at a point represented by a 3-dimensional vector in the Cartesian coordinate system. For example, the $m$-th transmitter is associated with a vector $\mathbf{c}_{t, m}=\left[x_{m}, y_{m}, z_{m}\right]$, and the $\mathrm{n}$-th receiver $\mathbf{c}_{r, n}=\left[x_{n}, y_{n}, z_{n}\right]$. For simplification, this paper considers only single point target case. And the target is assumed to be located at a general point $\mathbf{x}=[x, y, z]$ with constant velocity of $\mathbf{v}=\left[v_{x}, v_{y}, v_{z}\right]$. As the antennas are widely distributed, each of them will view the target with

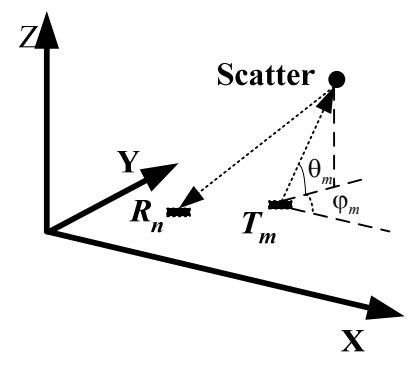

Figure 1. MIMO radar configuration. a different angle. Angle variables $\theta$ and $\varphi$ refer to the true elevation and azimuth as illustrated in Figure 1. We also assume that the phases and time at the transmitters and receivers are synchronized in advance. Meanwhile, the signal attenuation in different path is assumed to be the same.

Let $x_{m}(t)$ be the signal transmitted by the $m$-th transmitter that meets the requirement of narrow band assumption. It is expressed as

$$
x_{m}(t)=\exp \left(j 2 \pi f_{m} t\right) s_{m}(t)
$$

where $s_{m}(t)$ is the baseband waveform of the $m$-th transmitter. After the signal impinged back from the target to the $n$-th receiver, the echo is:

$$
e_{n}(t)=\sum_{m=1}^{M} \gamma x_{m}\left(t-\tau_{n m}\right) \exp \left(-j 2 \pi f d_{n m} t\right)
$$

where $\gamma$ is the complex reflection coefficient of the target, $\tau_{n m}$ is the round-trip delay, and $f d_{n m}$ is the Doppler frequency of the echo at the $n$-th receiver due to the $m$-th transmitter. We take the assumption that the target stops during the pulse transmission and reception. Then $\tau_{n m}$ is the round-trip delay at the start of observation time, and has the form

$$
\begin{aligned}
\tau_{n m}= & r_{T}^{m}(t) / c+r_{R}^{n}(t) /\left.c\right|_{t=0} \\
= & \sqrt{\left(x-x_{m}\right)^{2}+\left(y-y_{m}\right)^{2}+\left(z-z_{m}\right)} / c \\
& +\sqrt{\left(x-x_{n}\right)^{2}+\left(y-y_{n}\right)^{2}+\left(z-z_{n}\right)} / c
\end{aligned}
$$

where $c$ is the velocity of light in the media that the transmitters, receivers and targets are located in. In the case that the transmitters or the receivers are mounted on moving platform, the platform velocity can also be easily included in (3). For different scatter, $\tau_{n m}$ is a function of variables $x, y$, and $z$. Thus, through Taylor-series analysis at a reference point $\mathbf{x}_{0}=\left[x_{0}, y_{0}, z_{0}\right]$, (3) can be changed to

$$
\begin{aligned}
\tau_{n m}= & r_{T}^{m}(0) / c+r_{R}^{n}(0) /\left.c\right|_{[x, y, z]} \\
& \left(r_{T}^{m}(0) / c+r_{R}^{n}(0) /\left.c\right|_{\left[x_{0}, y_{0}, z_{0}\right]}\right)+ \\
& \left(\frac{\left(x-x_{0}\right)\left(\cos \theta_{m} \cos \varphi_{m}+\cos \theta_{n} \cos \varphi_{n}\right)}{c}+\right. \\
& \frac{\left(y-y_{0}\right)\left(\cos \theta_{m} \sin \varphi_{m}+\cos \theta_{n} \sin \varphi_{n}\right)}{c}+ \\
& \left.\frac{\left(z-z_{0}\right)\left(\sin \theta_{m}+\sin \theta_{n}\right)}{c}\right) \\
= & \tau_{n m}^{0}+\tau_{n m}^{\prime}
\end{aligned}
$$

where

$$
\tau_{n m}^{0}=r_{T}^{m}(0) / c+r_{R}^{n}(0) /\left.c\right|_{\left[x_{0}, y_{0}, z_{0}\right]}
$$

and 


$$
\begin{aligned}
& \tau_{n m}^{\prime}=\left(\frac{\left(x-x_{0}\right)\left(\cos \theta_{m} \cos \varphi_{m}+\cos \theta_{n} \cos \varphi_{n}\right)}{c}+\right. \\
& \frac{\left(y-y_{0}\right)\left(\cos \theta_{m} \sin \varphi_{m}+\cos \theta_{n} \sin \varphi_{n}\right)}{c}+ \\
& \left.\frac{\left(z-z_{0}\right)\left(\sin \theta_{m}+\sin \theta_{n}\right)}{c}\right) \\
& f_{d n m}=\frac{v_{x}\left(x-x_{m}\right)+v_{y}\left(y-y_{m}\right)+v_{z}\left(z-z_{m}\right)}{\lambda_{m} \sqrt{\left(x-x_{m}\right)^{2}+\left(y-y_{m}\right)^{2}+\left(z-z_{m}\right)^{2}}}+\frac{v_{x}\left(x-x_{n}\right)+v_{y}\left(y_{0}-y_{n}\right)+v_{z}\left(z-z_{n}\right)}{\lambda_{m} \sqrt{\left(x-x_{n}\right)^{2}+\left(y-y_{n}\right)^{2}+\left(z-z_{n}\right)^{2}}} \\
& =\frac{v_{x}\left(\cos \theta_{m} \cos \varphi_{m}+\cos \theta_{n} \cos \varphi_{n}\right)}{\lambda_{m}}+\frac{v_{y}\left(\cos \theta_{m} \sin \varphi_{m}+\cos \theta_{n} \sin \varphi_{n}\right)}{\lambda_{m}} \\
& +\frac{v_{z}\left(\sin \theta_{m}+\sin \theta_{n}\right)}{\lambda_{m}} \\
& f d_{n m}=\frac{1}{\lambda_{m}} \frac{d}{d t}\left(r_{T}^{m}(t)+r_{R}^{n}(t)\right)
\end{aligned}
$$

Again, through Taylor-series analysis (7) can be

It can be easily proved that (8) is a tantamount expression of conventional Doppler frequency of bistatic radar.

As each transmitting waveform is assigned to a distinct channel, orthogonality holds for all the transmitting signals. Thus, at each receiver, signals from $M$ transmit-

$$
\begin{aligned}
& y_{n m}\left(\mathbf{x}, \mathbf{x}_{0}, \mathbf{v}\right) \\
= & \gamma \exp \left(-j 2 \pi f_{m}\left(\tau_{n m}-\tau_{m m}^{0}\right)\right) \int_{-\infty}^{+\infty} s_{m}\left(t-\tau_{n m}^{0}\right) \exp \left(j 2 \pi f d_{n m} t\right) s_{m}^{*}\left(t-\tau_{n m}\right) d t+n_{n m}(t) \\
= & \gamma \exp \left(-j 2 \pi f_{m} \tau_{n m}^{\prime}\right) \mathbf{A}_{m m}\left(\tau_{m m}^{\prime}, f d_{n m}\right)+n_{n m}(t)
\end{aligned}
$$

where $\mathbf{A}_{m m}$ is the correlation between the $m$ - $t h$ transmitting waveform and its delay-Doppler shifted version, and $n_{n m}(t)$ is the noise component of the output.

Collectively, there are $N M$ outputs after matched filtering. By coherently summing all these outputs we can get:

$$
\mathbf{A}\left(\mathbf{x}, \mathbf{x}_{0}, \mathbf{v}\right)=\left|\sum_{n=1}^{N} \sum_{m=1}^{M} y_{n m}\left(\mathbf{x}, \mathbf{x}_{0}, \mathbf{v}\right)\right|^{2}
$$

Because both $\tau_{n m}^{\prime}$ and $f d_{n m}$ in (9) are affected by azimuth angles and elevation angels, the geometry configuration represented by a matrix $\mathbf{C}$ consisting of all the azimuth and elevation angels should be included in the ambiguity function. Ignoring the noise-based component and discarding the target reflection coefficient in (10), we can define the normalized ambiguity function for the proposed system as:

$$
\begin{aligned}
& \chi\left(\mathbf{x}, \mathbf{x}_{0}, \mathbf{v}, \mathbf{C}\right)=\frac{1}{M^{2} N^{2}} \\
& \left|\sum_{n=1}^{N} \sum_{m=1}^{M} \exp \left(-j 2 \pi f_{m} \tau_{n m}^{\prime}\right) \mathbf{A}_{m m}\left(\tau_{n m}^{\prime}, f d_{n m}\right)\right|^{2}
\end{aligned}
$$

As $\tau_{n m}^{\prime}$ and $f d_{n m}$ are related to $x-x_{0}, y-y_{0}, z-z_{0}, v_{x}, v_{y}$, $v_{z}$, azimuth angles and elevation angels, the range and ponents and geometry configuration can be assessed through the AF analysis. azimuth resolution as well as the effects of velocity com- ters can be firstly separated by down-converting into $M$ channels. Then, for each channel, a matched filter of corresponding transmitting waveform is employed at the interested range cell centered at $\mathbf{x}_{0}=\left[x_{0}, y_{0}, z_{0}\right]$. Thus, the $m$-th filter output at the $n$-th receiver can be expressed as
Besides, different waveforms may obtain different $\mathbf{A}_{m m}$, thus waveforms also play a key role in the MIMO radar ambiguity function. We take Linear Frequency Modulation (LFM) waveforms as an example to illustrate this point. A conventional LFM waveform defined by $u(t)=\operatorname{rect}(t / T) \exp \left(j \pi k t^{2}\right)$ has an correlation function like [12]:

$$
\mathrm{A}(\tau, v)_{L F M}=\exp \left(-j \pi k \tau^{2}\right)\left(1-\frac{|\tau|}{T}\right) \sin c\left((v T-B \tau)\left(1-\frac{|\tau|}{T}\right)\right)
$$

where $v$ is the Doppler frequency, $\tau$ is the time-delay, $k$ is the chirp rate, $T$ is the pulse width, $B$ is the bandwidth. From (12), it can be easily inferred that the bandwidth of single waveform adopted will impact the performance of the MIMO-HFSWR system.

\section{Resolution Capacity and PSL Performance}

In this section, the resolution capacity and PSL of the MIMO-HFSWR system using sparse frequency waveform are assessed by setting the Doppler frequency to zero in AF analysis. Sparse frequency waveforms consisting of stepped frequency linear frequency modulation signals are investigated. For simplification we just study a simplified 2-dimensional configuration. 


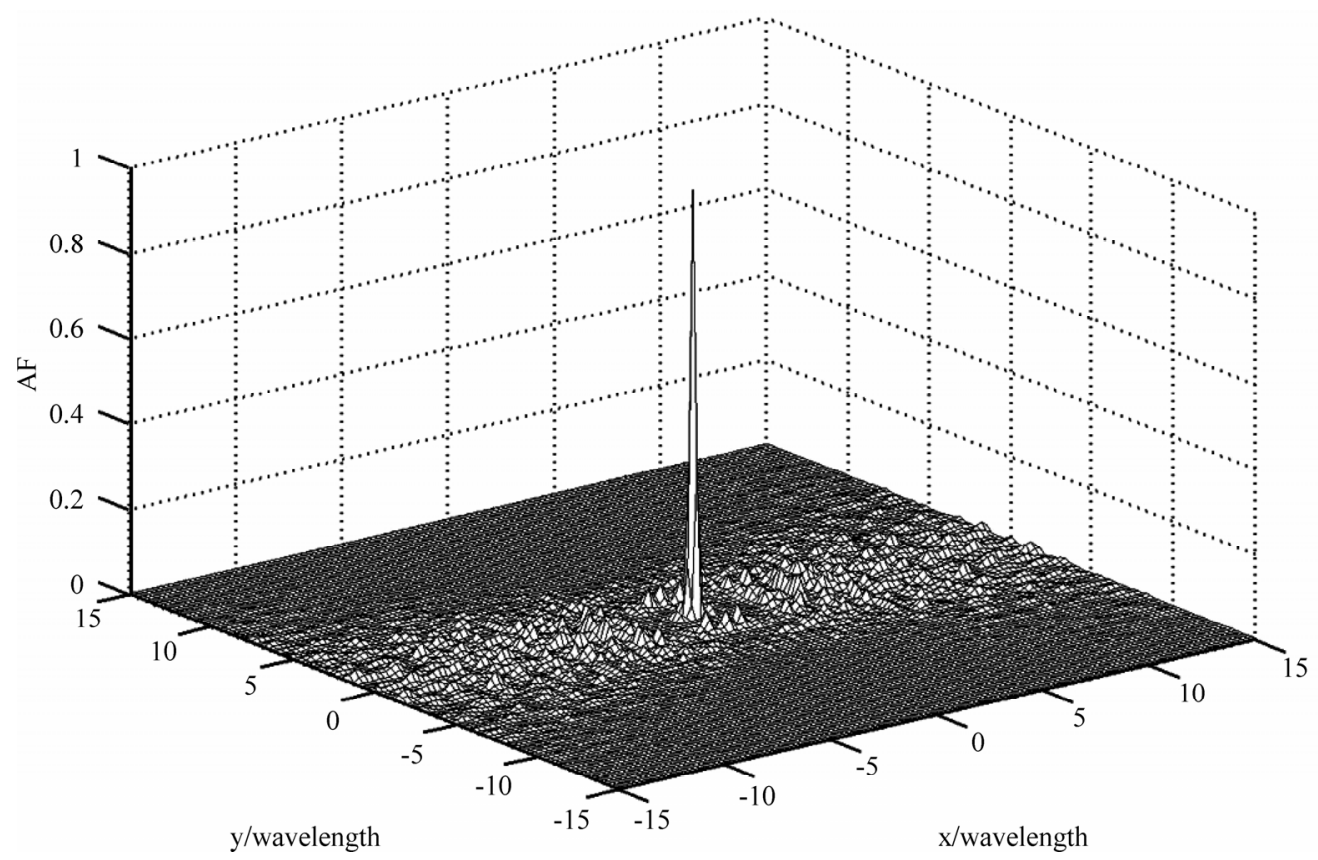

(a)

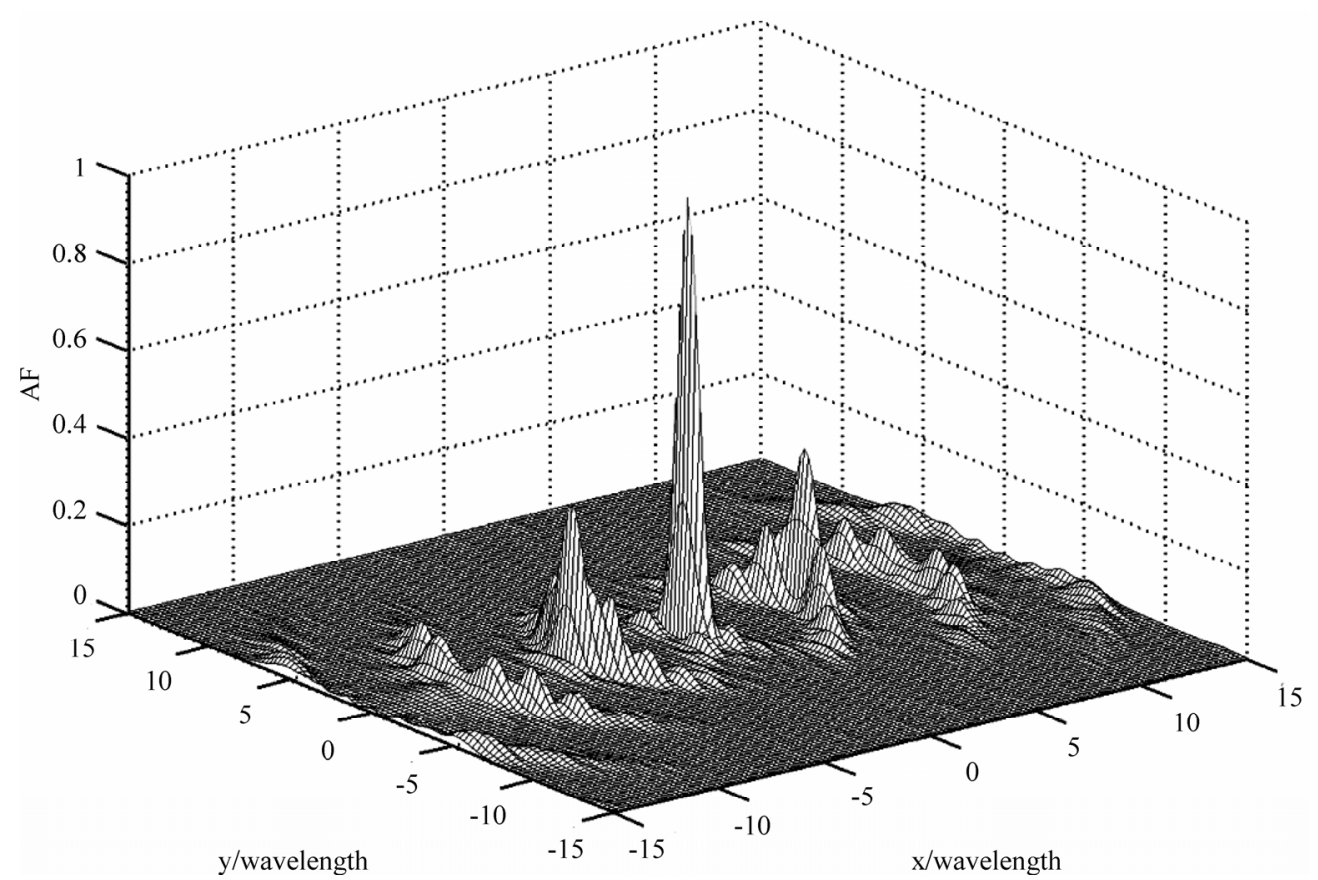

(b)

Figure 2. MIMO AF of (a) Sparse frequency waveforms (b) Single LFM signal, at zero Doppler frequency.

As we can see from above analysis, the ambiguity function of the proposed MIMO-HFSWR system depends on the system geometry configuration confined by all azimuth and elevation angles. Thus, we can ignore the true position of transmitters and receivers. We here take nine transmitters and nine receivers located evenly over spatial region of $(-\pi / 4, \pi / 4)$ for $\varphi$. Each transmitter will emit one LFM with an assigned start frequency. The pulse width is 100 us for all transmitters. The bandwidth of each LFM pulse is $500 \mathrm{kHz}$. The nine start frequencies of LFM waveforms are defined as the sequence of $\{5,6$, $7,8,9,8,7,6,5\} \mathrm{MHz}$. Orthogonality can be achieved 
by sequentially transmitting at transmitters or by setting the first five LFM waveforms to be up-chirps and the left four down-chirps [12]. We in this paper utilize the first mechanism. As a comparison, we also take an ambiguity function from a single LFM waveform with the same bandwidth and pulse width as well as the start frequency of $9 \mathrm{MHz}$. We also suppose to transmit it sequentially in time domain so that we can separate at each receiver the returns from different transmitters. By central coherent processing we can also get the results of AF as showed in Figure 2(b), which seems the same as that in [8].

The mesh plots of the AF are showed in Figure 2 and more details on resolutions and sidelobe characteristics are given in Figure 3 and Table 1. As is obvious from both Figure 3 and Table 1, the resolutions of MIMOHFSWR are at the level of one wavelength for both

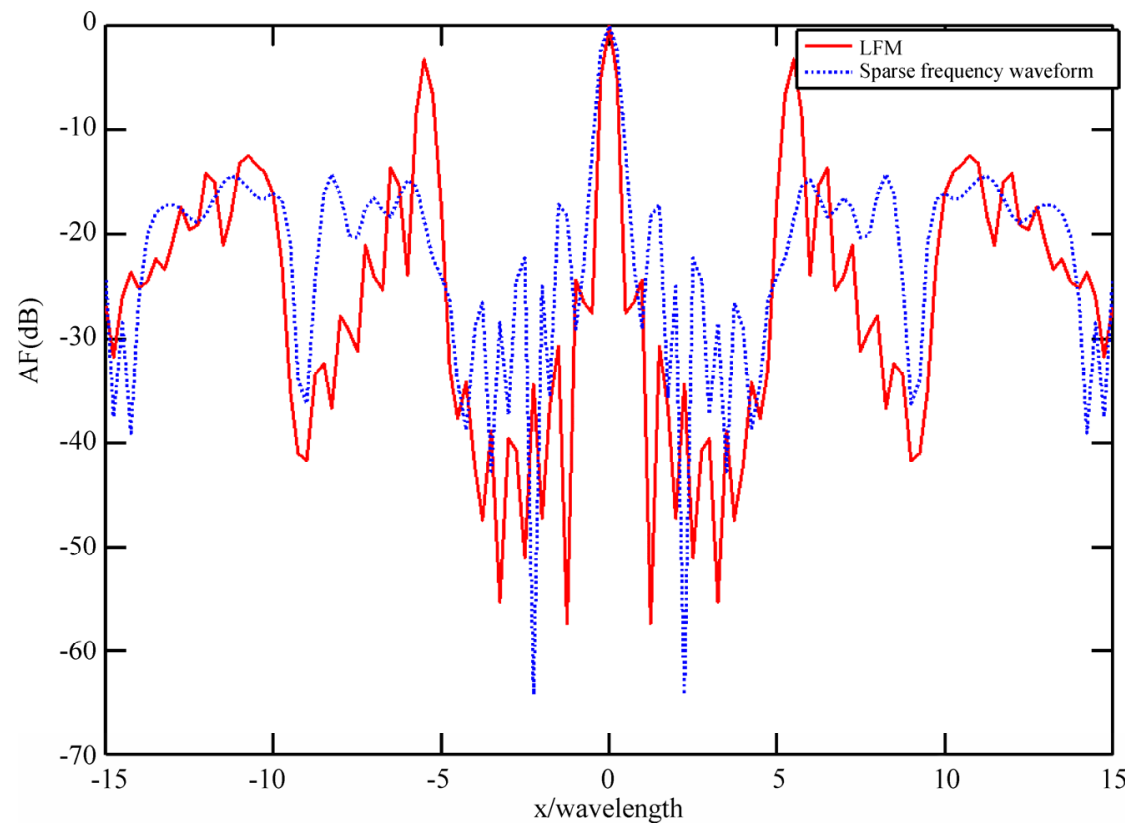

(a)

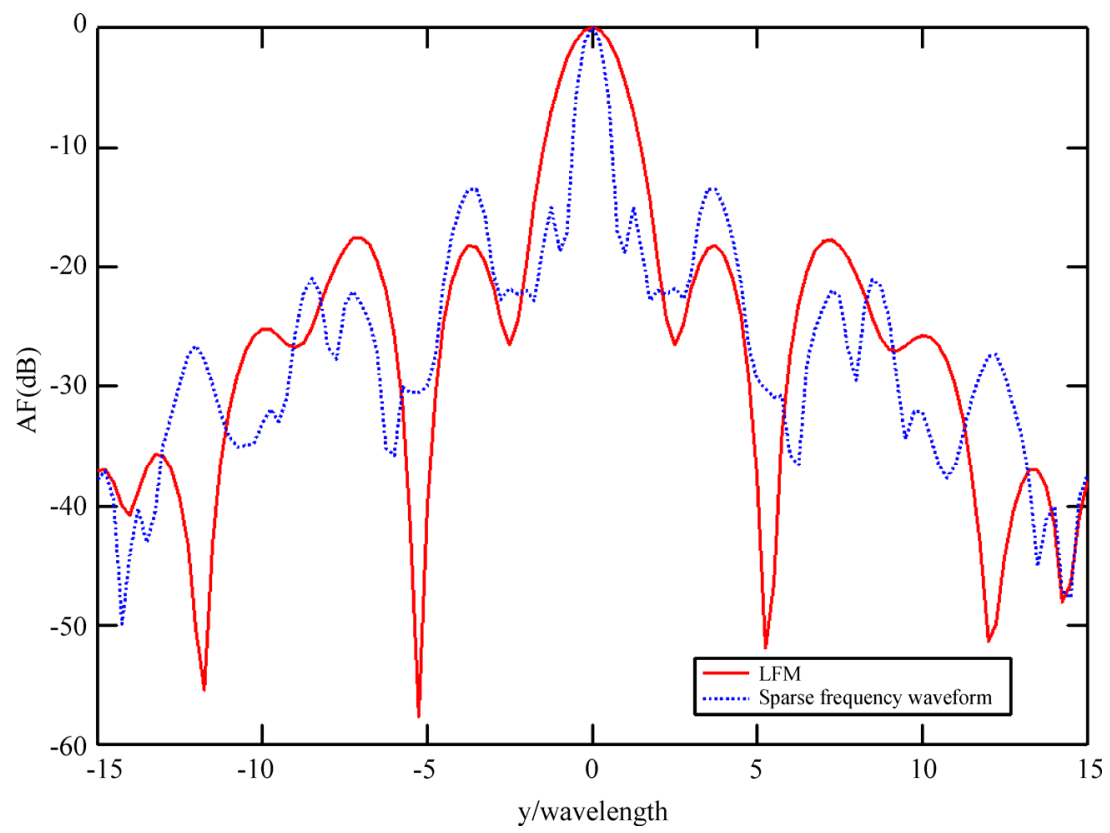

(b)

Figure 3. Resolution and sidelobe performances of sparse frequency waveforms (solid line) and single LFM signal (dotted line) along (a) $x$-axis and (b) $y$-axis. 
Table 1. Resolution and sidelobe characters of different waveforms.

\begin{tabular}{ccc}
\hline Item & Sparse frequency waveforms & LFM \\
\hline Resolution of $x$ & 0.31 wavelength & 0.58 wavelength \\
PSL-x & $-14.9 \mathrm{~dB}$ & $-3.2 \mathrm{~dB}$ \\
Resolution of $y$ & 0.9 wavelength & 1.6 wavelength \\
PSL-y & $-13.5 \mathrm{~dB}$ & $-16.5 \mathrm{~dB}$ \\
\hline
\end{tabular}

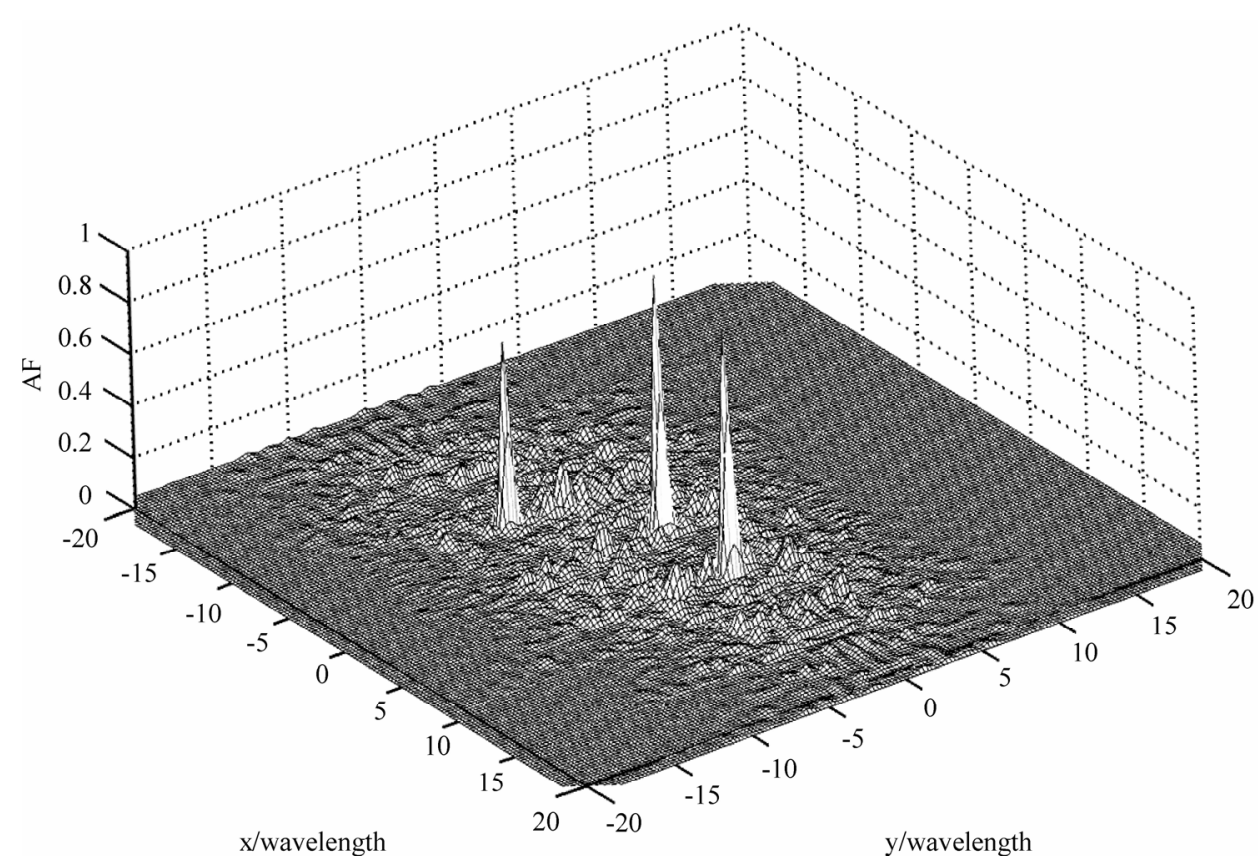

(a)

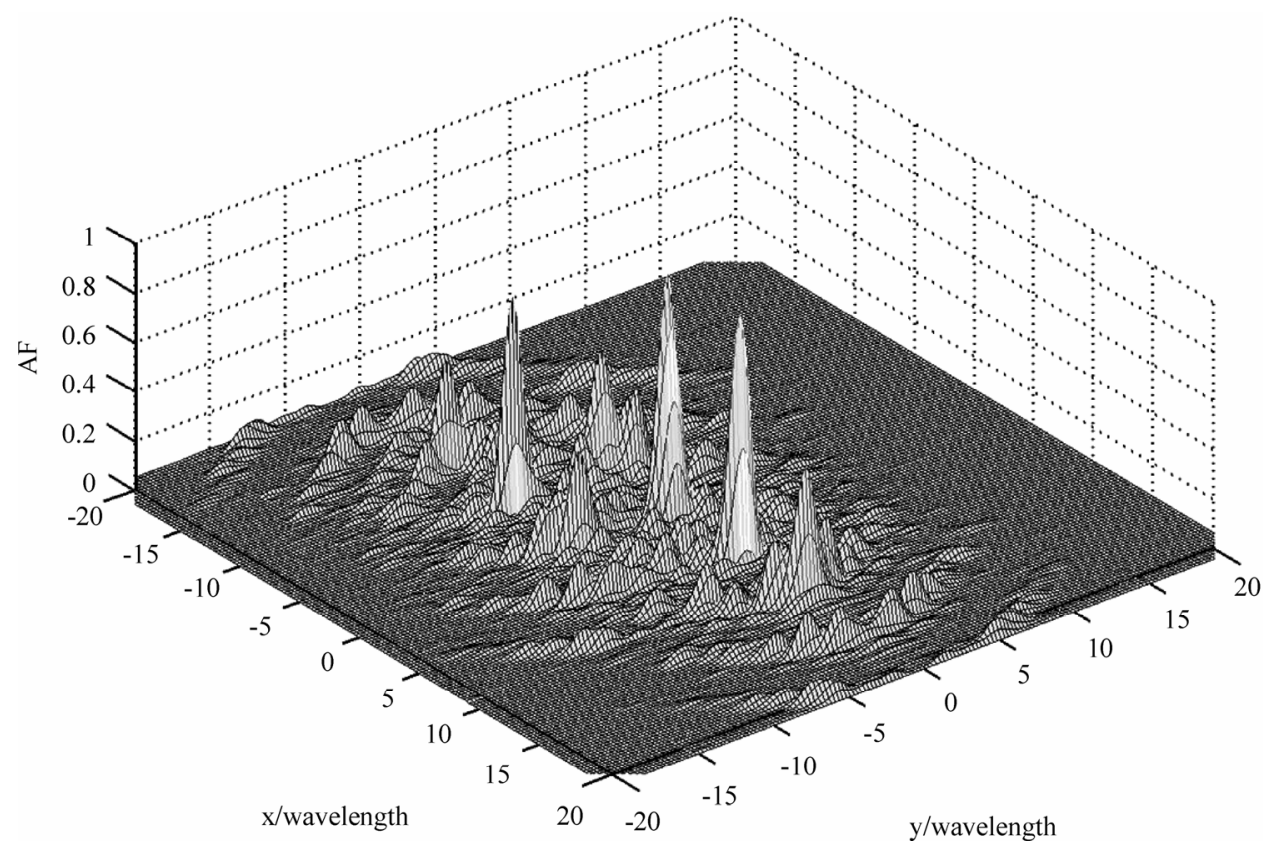

(b)

Figure 4. Resolution capacity in 3 point targets case (a) Sparse frequency waveforms (b) Single LFM waveform. 
sparse frequency waveforms and single LFM waveform. And the resolutions of sparse frequency waveforms are even better than those of common LFM signals. This is a great improvement for azimuth resolution and even for range resolution from conventional several kilometers to several tens meters. Meanwhile, as HFSWR always works in a highly congested spectrum environment, the sparse frequency waveform approach can provide better flexibility on choosing available channels than wave- forms confined in only one channel. The sidelobes of $\mathrm{x}$-axis and $\mathrm{y}$-axis are well below -13 and $-14 \mathrm{~dB}$ for sparse frequency waveforms, respectively, which is a significant improvement compared with the side lobe level from the single LFM waveform within the same channel. It demonstrates that the sidelobe levels can be suppressed by frequency diversity in random arrays [13]. This is another advantage of sparse frequency waveform.

Multiple targets case are illustrated in Figure 4, where

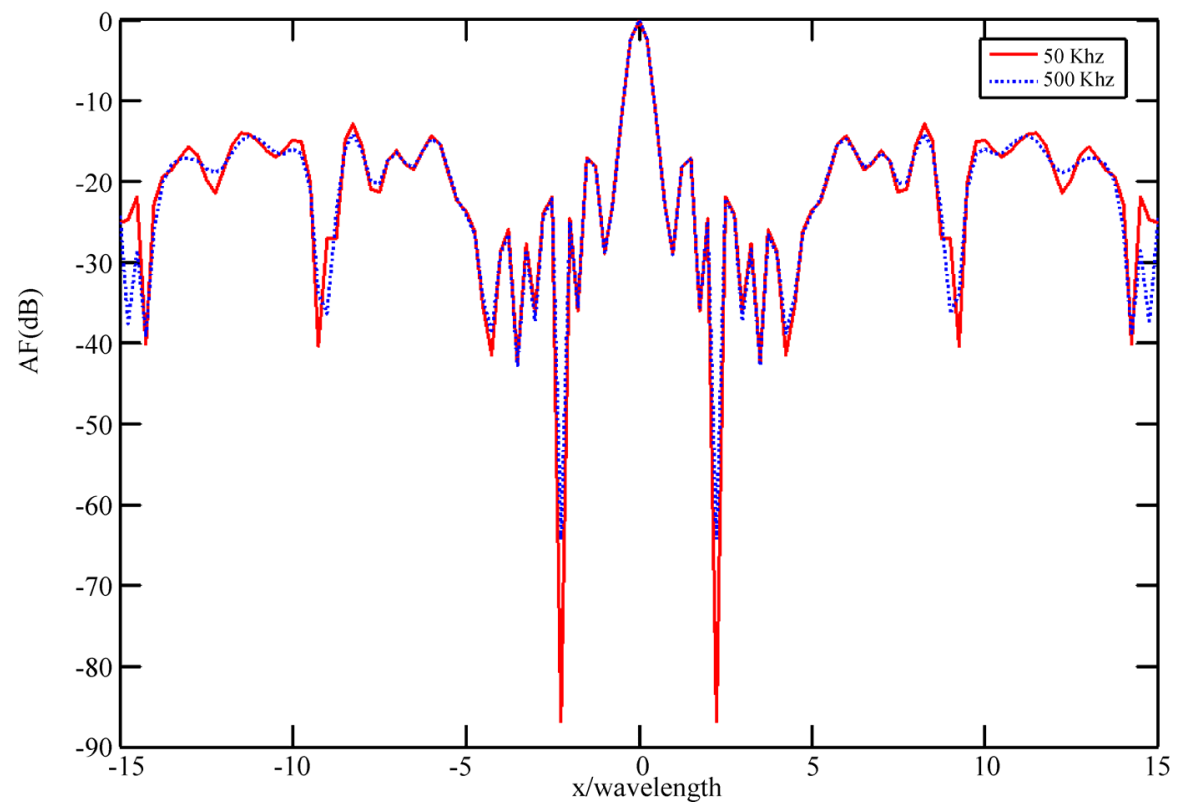

(a)

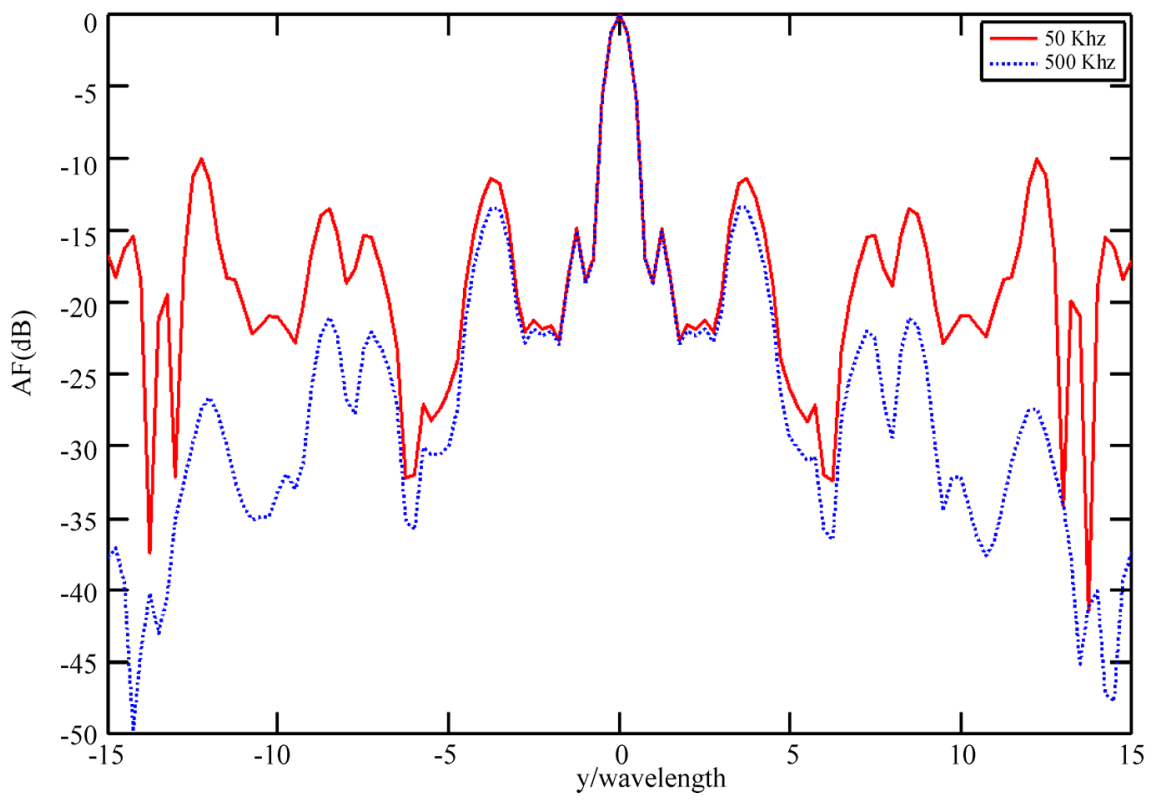

(b)

Figure 5. Bandwidth effect on resolution and sidelobe performance along (a) $x$-axis and (b) $y$-axis. Solid line is associated with bandwidth $50 \mathrm{KHz}$, while the dotted line is $500 \mathrm{KHz}$. 


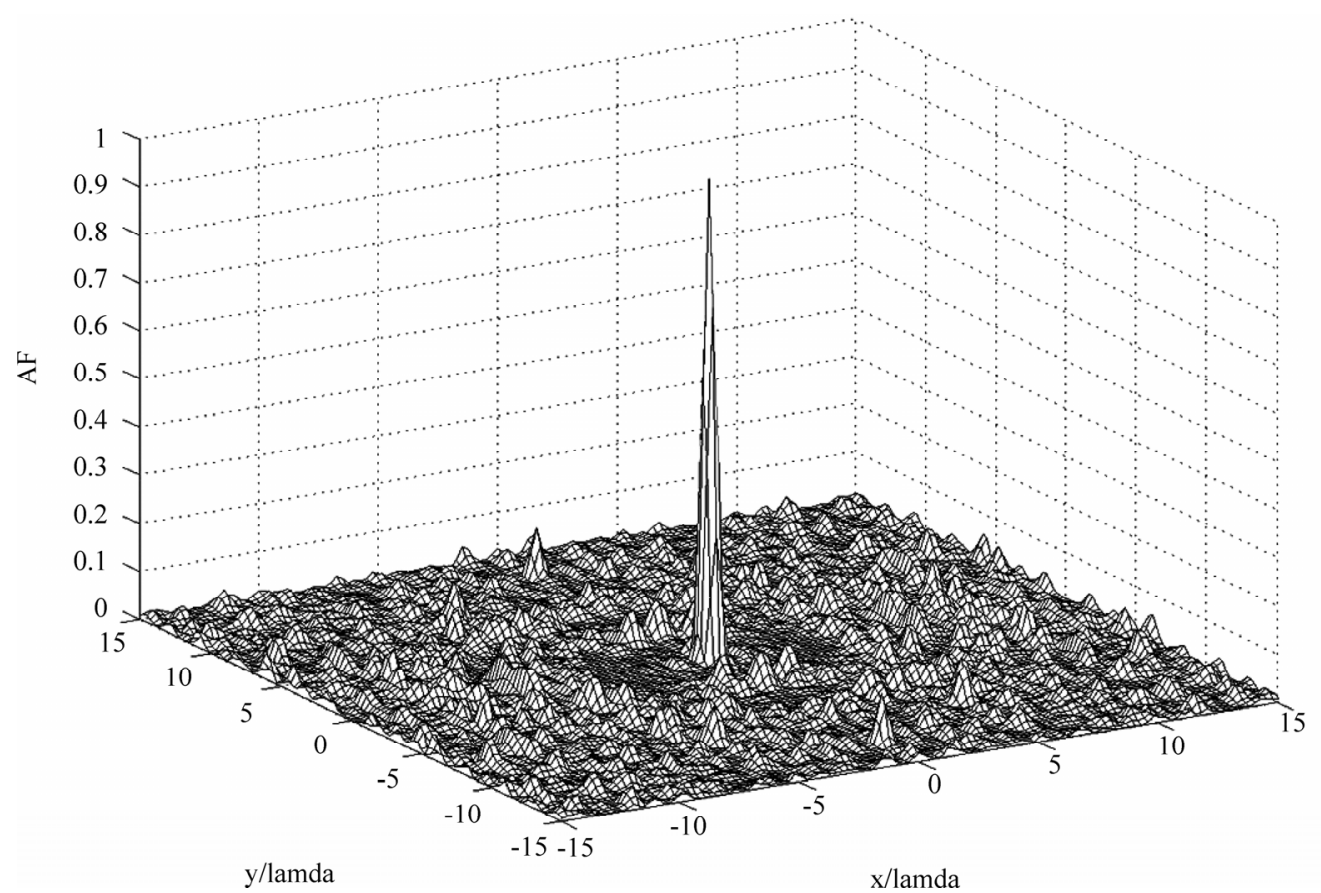

(a)

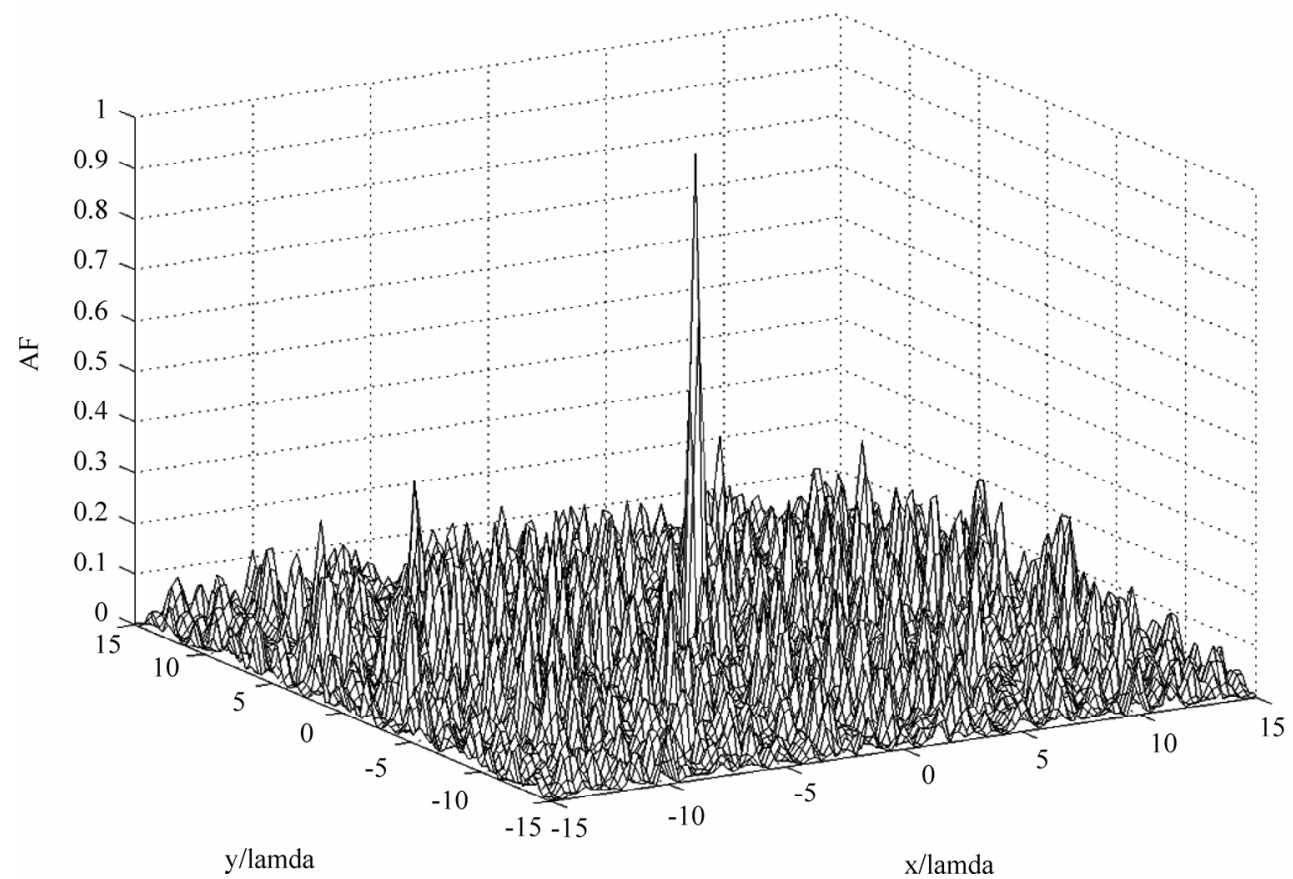

(b)

Figure 6. Ambiguity functions of (a) Configuration 1 and (b) Configuration 2 in case 1 with velocity $\left[v_{x}, v_{y}\right]=[500,500] \mathrm{m} / \mathrm{s}$. Configuration $1(9 \times 9)$, Configuration $2(5 \times 5)$, both evenly distributed in $(-\pi / 4, \pi / 4)$.

three targets are located in $[0,0],[0,10]$, and $[-10,-10]$. The coordinate system is expressed in multiples of wavelength. As we can see, by using sparse frequency waveform set, the system can better distinguish different targets than by using waveform set in the same channel.
Bandwidth effect is illustrated in Figure 5. We take a set of sparse waveforms like that mentioned above. The difference is that the bandwidth is $50 \mathrm{KHz}$ for each waveform. From Figure 5 we can see that even with smaller bandwidth, the resolution capacity is not much impacted. 


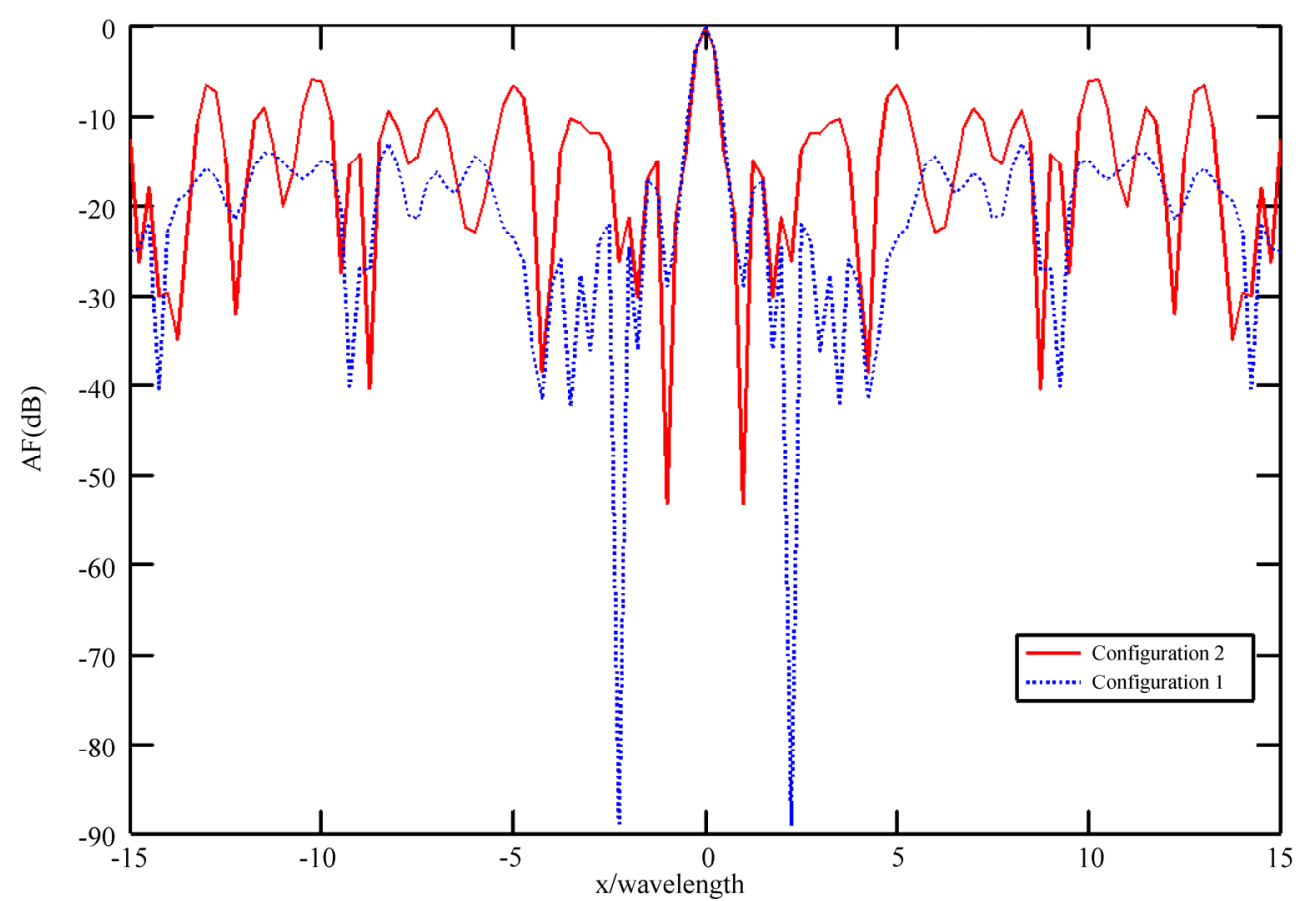

(a)

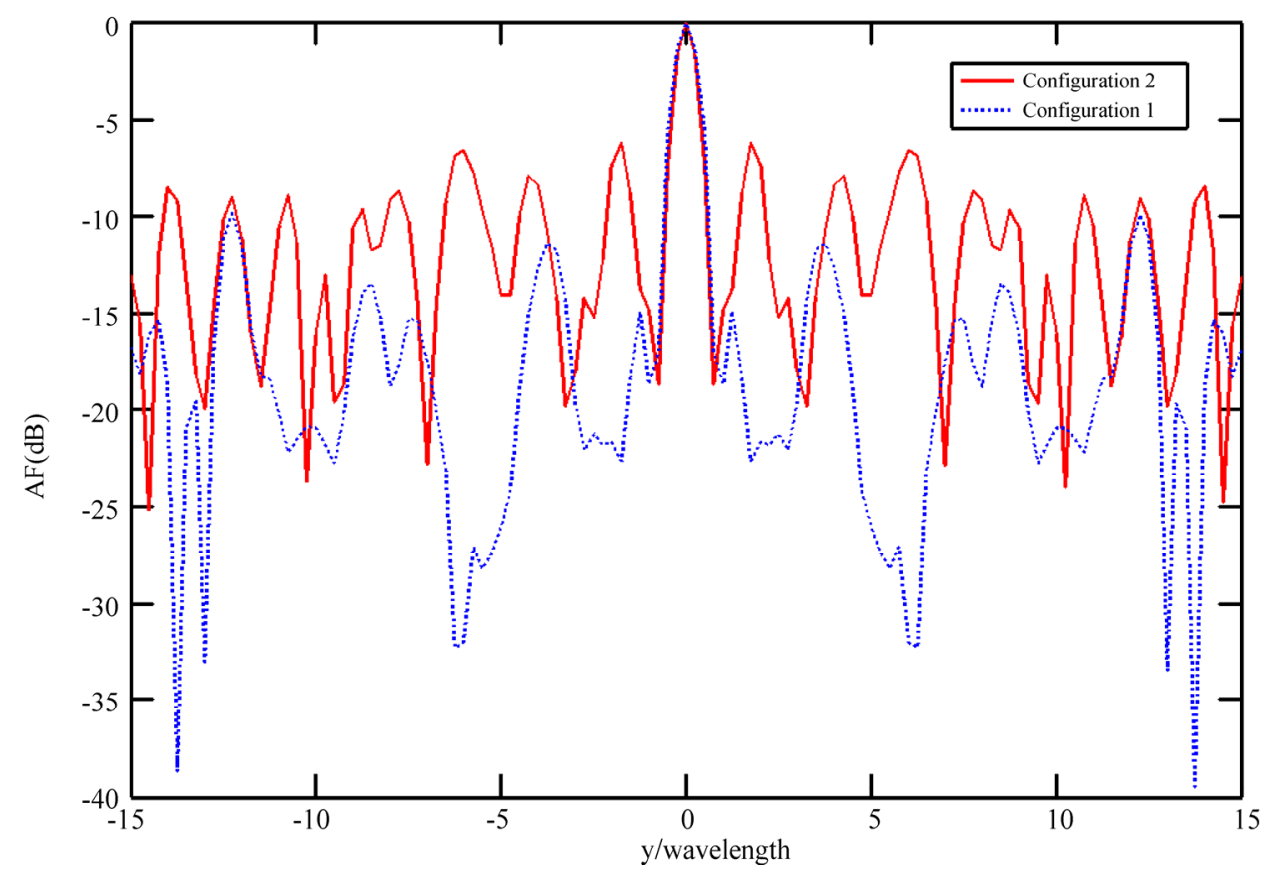

(b)

Figure 7. Resolution and sidelobe performance along (a) $x$-axis and (b) y-axis in case 1 with velocity $\left[v_{x}, v_{y}\right]=[500,500] \mathrm{m} / \mathrm{s}$. Configuration $1(9 \times 9)$, Configuration $2(5 \times 5)$, both evenly distributed in $(-\pi / 4, \pi / 4)$.

However, the PSL performance is deteriorated. The PSL in $x$-axis is about $-12.9 \mathrm{~dB}$ and is about $-10 \mathrm{~dB}$ in $y$-axis for this waveform set. Thus, we can see that the larger the bandwidth adopted, the lower the sidelobes in both $x$-axis and $y$-axis.
In this case study, the simulation results demonstrate that MIMO-HFSWR with sparse frequency waveform has superior resolution than conventional HFSWR in both range and downrange domain. Sidelobe levels can be suppressed by using sparse frequency waveforms. 
Further work will be focused on the suppression of sidelobe levels by waveforms with effective frequency diversity scheme.

\section{Doppler and Geometry Factor}

As HFSWR is always operated in Doppler circumstances, the AF with Doppler effects should be further investigated. Meanwhile, unlike monostatic radar the distributed MIMO radar is confined by the geometry configuration. Thus the geometry factor should also be investigated. Two cases are given below to investigate the Doppler and configuration effect.

In Case 1, we study the configuration effect. Target of this case is with velocity of $\left[v_{x}, v_{y}\right]=[500,500] \mathrm{m} / \mathrm{s}$. This is a high velocity target case corresponding to air targets. There are two configurations. For Configuration 1, in the region of $(-\pi / 4, \pi / 4)$ there are nine transmitters and nine receivers, evenly distributed. The transmitting waveforms are defined as those in Section 3 except that each one has $10 \mathrm{kHz}$ bandwidth. For practical HFSWR application, only a limited number of continuous clear channels with bandwidth of a few kilo-Herz in the $3-30 \mathrm{MHz}$ high frequency band can be found and used at a time when interference is considered [1,2]. Thus, $10 \mathrm{kHz}$ bandwidth is used for a much more similitude in real condition of the HFSWR system. For Configuration 2, in

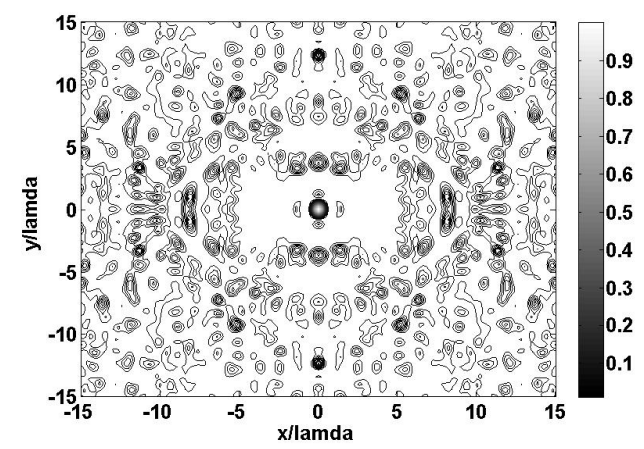

(a) $\mathrm{v}=[0,0] \mathrm{m} / \mathrm{s}$

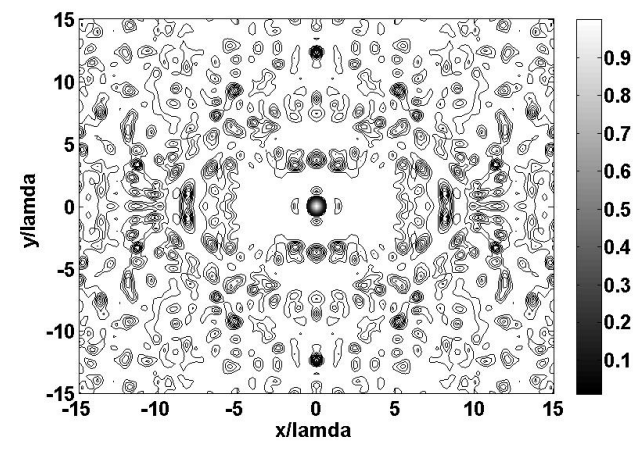

(c) $\mathrm{v}=[-10,5] \mathrm{m} / \mathrm{s}$ the same region of $(-\pi / 4, \pi / 4)$ there are five transmitters and five receivers, evenly distributed. The waveforms are the first five used in Configuration 1 of Case 1. Thus, the total spectra employed by these two configurations are the same. As illustrated in Figure 6, Figure 7, both configurations in this case show high resolution capabilities. However, Configuration 1 with more transmit-receive pairs shows better sidelobe performance in both $\mathrm{x}$-axis and y-axis. The PSL in $x$-axis is about $-12 \mathrm{~dB}$ and is about $-10.5 \mathrm{~dB}$ in $y$-axis for Configuration 1. Based on our numerous simulation experiments, it is found that as more pairs of transmitter and receiver are set in a much wider spatial region, the resolutions can be slightly improved and the PSLs of $y$-axis and x-axis can be further reduced. However, systematic study on the PSLs reduction through geometry optimization will be explored in the future.

In Case 2: we have four velocity settings like $[0,0]$ $\mathrm{m} / \mathrm{s},[100,-100] \mathrm{m} / \mathrm{s},[-10,5] \mathrm{m} / \mathrm{s}$, and $[500,500] \mathrm{m} / \mathrm{s}$. The geometry configuration in Case 2 is the same as Configuration 1 in Case 1. We also take the waveform set of Configuration 1 of Case 1 in this case study. Figure 8 shows the results of Case 2. We can see from Figure 8 that the proposed system shows similar characteristics in different Doppler context, which means the resolution and PSL performance are both insensitive to Doppler frequency. Thus, for both high speed air targets and low

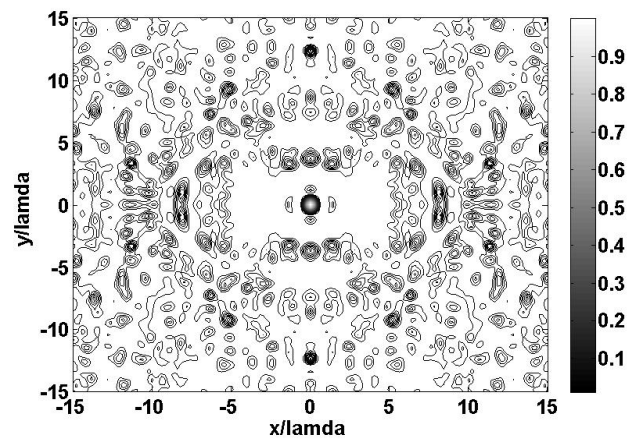

(b) $\mathrm{v}=[100,-100] \mathrm{m} / \mathrm{s}$

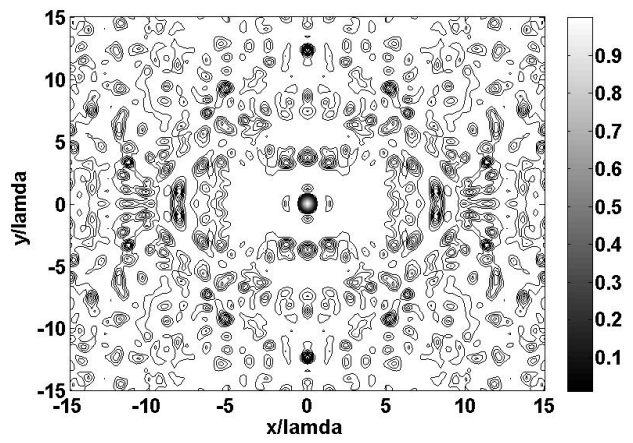

(d) $\mathrm{v}=[\mathbf{5 0 0 , 5 0 0 ]} \mathrm{m} / \mathrm{s}$

Figure 8. Ambiguity functions of different velocity with Configuration 1. 
velocity surface targets, the proposed system can also have high resolution performance.

\section{Conclusions}

In this paper, the concept of distributed MIMO-HFSWR radar transmitting sparse frequency waveforms is proposed. The AF of this proposed system is derived in detail. Potential advantages of the proposed system on resolution capacity and PSL performance are assessed through AF analysis and simulations. The impacts of Doppler effects and the geometry configuration factor are also studied. It has been found that the system has several distinguished characteristics. Firstly, the range resolution and the azimuth resolution can be improved to the level of one wavelength, namely, only tens meters and the PSL is reduced to a much lower level with sparse frequency waveforms. Meanwhile, the resolutions are not restricted by individual bandwidth while the PSL can benefit from large bandwidth. Secondly, the performance of fine resolution and low PSL are insensitive to the Doppler effects. Thus, for both high speed air and low velocity surface targets, the proposed system also has high performance. Thirdly, the resolution capacity and PSL performance can be optimized through geometry configuration optimization. In addition, multistatic configuration provides large flexibility to find a proper place to locate the radar transmitters and receivers; by using sparse frequency waveforms, it is much easier to find more available channels in different locations thus the co-channel interference can be avoided and the performance can be further improved. Further studies will be conducted on the surveillance strategy and high quality waveforms with better AF performance. Meanwhile, synchronization problem should also be paid special attention to so that coherent processing can be conducted perfectly.

\section{References}

[1] H. W. H. Leong and B. Dawe, "Channel availability for east coast high frequency surface wave radar systems," Defence R\&D Canada, Technical Report, DREO TR 2001-104, November 2001.
[2] R. J. Riddolls, "A Canadian perspective on high frequency over-the-horizon radar," Defence R\&D Canada, Technical Report, DREO TR 2006-285, December 2006.

[3] D. W. Bliss and K. W. Forsythe, "Multiple-input multiple-output (MIMO) radar and imaging: Degrees of freedom and resolution," Proceedings of the 37th Asilomar Conference on Signal, Systems and Computers, pp. 54-59, November 2003.

[4] J. Li, "MIMO radar: Diversity means superiority," Proceedings of the 14th Annual Adaptive Sensor Array Processing Workshop-2006, June 6-7, 2006.

[5] S. Peter, J. Li, and Y. Xie, "On probing single design for MIMO radar," IEEE Transactions on Signal Processing, Vol. 55, No. 8, pp. 4151-4161, August 2007.

[6] G. San Antonio, D. R. Fuhrmann, and F. C. Robey, "MIMO radar ambiguity function," in IEEE Journal of Selected Topics in Signal Processing, Vol. 1, No. 1, pp. 167-177, June 2007.

[7] E. Fisher, A. Haimovich, R. Blum, L. Cimini, D. Chizhik, and R. Valenzuela, "Spatial diversity in radars - models and detection performance," IEEE Transactions on Signal Processing, Vol. 20, No. 3, pp. 823-838, March 2006.

[8] N. H. Lehmann, A. M. Haimovich, R. S. Blum, and L. Cimini, "High resolution capabilities of MIMO radar," Proceedings of the 40th Asilomar Conference on Signal, Systems and Computers, pp. 25-30, November 2006.

[9] D. R. Kirk, J. S. Bergin, P. M. Techau, and J. E. Don Carlos, "Multi-static coherent sparse aperture approach to precision target detection and estimation," Proceedings of 2005 IEEE Radar Conference, pp. 579-584, May 2005.

[10] G. H. Wang, W. X. Liu, and Y. L. Lu, "Sparse frequency transmit waveform design with soft power constraint by using PSO algorithm," Proceedings of 2008 IEEE Radar Conference, pp. 1-6, May 2008.

[11] W. X. Liu, Y. L. Lu, and M. Leisturgie, "Optimal sparse waveform design for HFSWR system," Proceedings of 2007 International Waveform Diversity and Design Conference, pp. 127-130, May 2007.

[12] N. Levanon and E. Mosezen, Radar Signals, John Wiely \& Sons, New Jersy, 2004.

[13] B. D. Steinberg and E. H. Attia, "Sidelobe reduction of random arrays by element position and frequency diversity," IEEE Transactions on Signal Processing, Vol. 31, No. 6, pp. 922-930, November 1983. 